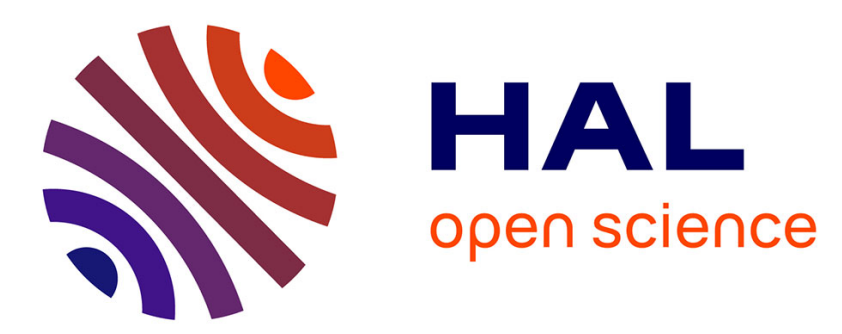

\title{
Virtual reality coupled with adapted physical interface for a better evaluation of the innovative surgical instrument
}

Duy Minh Phan Nguyen, Jérôme Tonetti, Guillaume Thomann

\section{- To cite this version:}

Duy Minh Phan Nguyen, Jérôme Tonetti, Guillaume Thomann. Virtual reality coupled with adapted physical interface for a better evaluation of the innovative surgical instrument. 23th CIRP Design Conference, Mar 2013, Bochum, Germany. 10 p. hal-00903913

\section{HAL Id: hal-00903913 \\ https://hal.science/hal-00903913}

Submitted on 13 Nov 2013

HAL is a multi-disciplinary open access archive for the deposit and dissemination of scientific research documents, whether they are published or not. The documents may come from teaching and research institutions in France or abroad, or from public or private research centers.
L'archive ouverte pluridisciplinaire HAL, est destinée au dépôt et à la diffusion de documents scientifiques de niveau recherche, publiés ou non, émanant des établissements d'enseignement et de recherche français ou étrangers, des laboratoires publics ou privés. 


\title{
Virtual reality coupled with adapted physical interface for a better evaluation of the innovative surgical instru- ment
}

\author{
D. M. Phan Nguyen ${ }^{1}$, J. Tonetti ${ }^{2}$, G. Thomann ${ }^{1}$ \\ ${ }^{1}$ G-SCOP Laboratory, Grenoble Institute of Technology, France \\ ${ }^{2}$ Orthopaedic and traumatology centre, Michallon Hospital, Grenoble, \\ France \\ Nguyen-Duy-Minh.Phan@gi-etu.grenoble-inp.fr \\ guillaume. thomanneg-scop.inpg.fr \\ JTonetti@chu-grenoble.fr
}

\begin{abstract}
In the domain of designing innovative products in the medical field, investigations are often oriented towards communication between actors and needs comprehension. In the DESTIN (DEsign of Surgical/Technological INnovation) project, User Centered Design methodology with concrete experiments is applied. Researchers propose emulations in an operating room for coevaluation of innovative products and new adapted surgical procedures. In this paper, they intend to evaluate the usage of the product in a virtual environment using a 3D haptic feedback system. Researchers not only propose a better ergonomic situation of the physician in front of the operating screen, but also increase the performance of the simulator in order to allow the manipulation of the innovative surgical instrument developed. We used virtual reality environment and the manufactured prototype with the aim to validate the new surgical procedure and the innovative designed surgical instrument.
\end{abstract}

Keywords: Virtual Reality, 3D-Haptic feedback system, Prototyping, User Centered Design (UCD), Minimally Invasive Surgery

\section{Introduction}

The development of new technologies in medicine can significantly improve the effectiveness. On the contrary, the use of more complex systems tends to make the practice of medicine more difficult. In particular, this complexity reinforces the importance of preoperative planning and postoperative monitoring. New technologies in informatics and virtual reality allow physicians to better interpret the enormous amount of information that is provided by the imaging systems or therapy systems [1]. Specifically, virtual reality allows better understanding, better planning and better work through visualization of three-dimensional images of anatomy and pathology. In addition, virtual reality supports the practitioner through the stages of diagnosis, therapy, and postoperative monitoring.

The main aim of DESTIN project (Design of Surgical-Technological INnovation) is to propose a new methodology focused on this specific context: How to create a

adfa, p. 1, 2011

(C) Springer-Verlag Berlin Heidelberg 2011 
new operative surgical procedure and innovative surgical instrument when a new medical approach is imagined?

The specific surgical application we are working on addresses thoraco-lumbar fracture. The current "classical" procedure is carried out with the patient in the prone position under general anesthesia. The surgeon performs a posterior open approach through a $15 \mathrm{~cm}$ large incision. The posterior vertebral arch is exposed. Pedicle screw entry points are chosen by direct visual control and they are fixed to the vertebrae. Rods are placed to connect the pedicle screws together. Prone placement added with rod-screw connection provides reduction of the trauma deformity and durable stability Thus, vertebrae are preventing from moving while bone healing and graft fusion takes place.

The new surgical procedure proposed by the surgeon consists in inserted the rod inside the pedicular screw in MIS (Minimally-Invasive Surgery). Thus, new little incisions should allow the insertion of the rod in the three pedicle screws.

Currently, researchers, designers and the medical staff regularly work in the real operating room. This work was very effective but necessitates heavy organization and management (mainly in the hospital), the creation of mannequins, the manufacturing of many prototypes, etc.

To facilitate this procedure, we create a CATIA CAD model of the virtual operating room. It integrates patient, medical equipment and surgical instruments. In this virtual environment, the surgeon has to manipulate the virtual surgical instrument on the virtual patient's spine (the spine has been modeled in a compatible format as the CATIA environment and integrated in a mannequin placed on the operative table). The goal of this exercise is to provide information to the designers for the validation of the innovative surgical instruments during the design process. At the same time, it also allows surgeons to perform the operative procedures with haptic feedback as in the real operative case.

The difficulty in our research concerns the ability to sufficiently represent the virtual environment for the co-validation of the medical procedure and the innovative surgical instrument.

Knowing that surgeons can already manipulate the virtual innovative surgical instrument using a 3D-Haption(C) haptic system in the virtual operating room, the research questions can be summarized as follow:

- How to modify the configuration of the virtual reality room and the physical interface for a better integration of the physician in the virtual environment?

- Which are the optimal dimensions of the virtual surgical instruments for a best representation and manipulation of the real surgical intervention using the 3D-Haption (C) haptic system?

To answer these questions, this research methodology is proposed:

- To research some ergonomic references in the surgery domain and compare them to our virtual reality room organization,

- Secondly to design and to link a new physical interface to the arm of the 3DHaption(C) haptic system,

- To modify the virtual model and to compare the surgical intervention feasibility with the real one. 
In this article, we firstly present the User Centered Design methodology we use during our study. Then we focus on the virtual reality and ergonomic applications and research in the surgical field. This first step allows us to analyze the general situation in the world. From this work, we propose modifications and adaptations of our current virtual operating room and 3D-Haption (C haptic system user interface.

Next, we present the results of the manipulation in virtual environment and conclusions concerning its efficiency related to the situation in real situation.

\section{User Centered Design}

User Centred Design (UCD) is considered as one of the cornerstones theories about user involvement. UCD, as a design approach, was first time introduced in NF EN ISO 9241-210: Human-Centred Design Processes for Interactive Systems [2]. The main issue is how to involve, integrate and consider the end-user and its requirements throughout the product design process. This ISO 13407 model proposes technical points the project must encompass to be considered as human centred: 1 - a certain knowledge of the end-users: their tasks and of their environment -2 - an active participation of these end-users, the clear understanding of their needs and the requirements linked with the tasks $-3-$ an appropriate distribution of the endusers/technological functions -4 - an iterative design solution $-5-$ the intervention of a multidisciplinary designing team. This is necessary to better interpret the enduser, its knowledge and how-know: human factors, information architecture, design, quality, marketing, etc.

The UCD cycle is decomposed into six main steps (Figure 1). It is an iterative cycle (step 2 to 5) which ends when the system answers the end-user requirement (step 6).

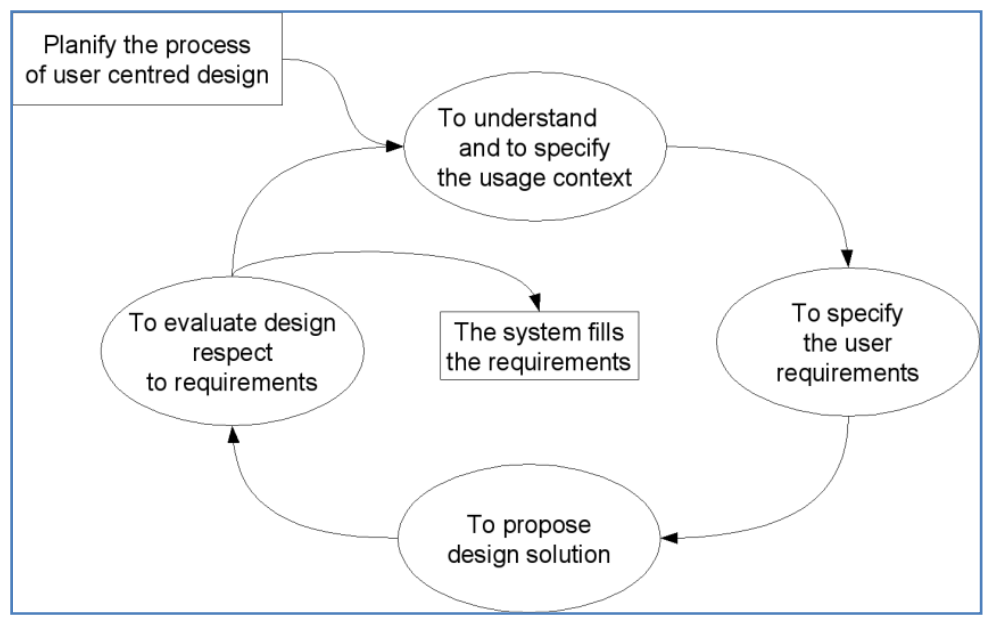

Fig. 1. The six steps of the UCD cycle

To better understand this UCD design steps, Jokela et al. propose another interpretation of this NF EN ISO 9241-210 UCD Process. They explain more concretely how it can be applied on a project and suggest a new UCD process model [3]. Another 
important issue in UCD is how identifying and selecting relevant end-users in the development work. In practice it is commonly possible to involve only a limited number of users, and therefore it is very important to define criteria in order to select the most "representative users" to centre the design on their requirements and expectations

\section{Virtual reality, ergonomics and their application in surgical field}

\subsection{Virtual reality and application to the surgical field}

Virtual Reality (VR) is an interactive immersive data-processing simulation in real or imaginary environments. Currently, the technology of RV was applied in many different fields such as: formation by simulator (driving vehicles, aerospace), design of products, the simulation of surgery, meteorology...

In the surgical field, the laparoscopy is a new procedure which requires surgeons to observe the operations on a monitor and requires acquisitions of new competences. This Minimally Invasive Surgery (MIS) differ from the opened surgery by the fact that the surgeon operates through small incisions and uses specific instruments as scalpel, grips, nets, etc. [4]. In spite of its many advantages (faster recovery of the patients, less damage with healthy tissues and smaller scars, less pain and less need for drugs), the MIS requires a long time training eyes-hands coordination.

Researches developing the haptic control feedback device can be found in [5-7]. To follow the user intentional movements, by interaction between hand and device, high powerful haptic devices must be able to produce force feedback. Consequently, it is essential to closely examine the human touched and the constraints of application during the construction of these devices. A haptic interface with 4 degrees of freedom was designed by Guiatni et al. to compare it with devices commercially available [4]. This device has the capacity to offer force feedback in all the degrees of freedom available during the MIS procedure.

In our case, researchers, designers and physicians work together on the development of a virtual environment to simulate a MIS operation on the spinal column. The goal is to create an integral virtual surgical environment with surgical instruments and haptic feebdack in a model of the operating room.

\subsection{Ergonomics in the surgical field}

Ergonomics is a relatively new science. It is based on the models in the design of machines and tools that optimize the performance of users. In order to improve the simulation conditions in virtual reality surgery environment that create the better immersion for surgeons who will eventually be the primary user of this innovative surgical instrument, we had to first study some articles to find the factors that influenced the comfort of surgeons during operations in the operating room. The optimum ergonomic position of the monitor was defined according to various sources in the literature [8], [9], [10], [11]. The monitor was at a distance of $0.6 \mathrm{~m}$ apart from the subjects' eyes. The monitor height (from the middle of the screen to the ground) was 
between the operating surface and eyelevel height, and the monitor was inclined (to a maximum of $15^{\circ}$ ) as by the subjects.

The optimal operating surface height was $80 \%$ of the elbow height and the table was positioned in $20^{\circ}$ tilt. [12].

In an article submitted by Gurvinder Kaur [13], he conducted a test to find the height of the ergonomics table in the minimally invasive surgery. This study aims to propose an ergonomic table height required for the surgeon height so they can perform comfortably in the operation. In this study, the height of the table has an effect on the upper joint movements of the shoulders, arms and wrist during laparoscopy. Table height should vary from 65 to $90 \mathrm{~cm}$ from the floor. The surgeon should be able to adjust the table corresponding to his/her height in order to bring upper joint movements to the minimum position with the resultant less discomfort in the shoulder, back elbow and the wrist. After analyzing the ratio between the surgeon's height with the height of the operating table, it was assumed that the height of the operating table should be calculated as follows:

Table Height $=$ Surgeon's Height X 0.49

The ideal posture for the MIS is supposed in the literature [14] and [15]. The arms are slightly removed, retroversion, and turned inward at the level of the shoulder (abduction $<30^{\circ}$ ). The elbows are bent at about $90-120^{\circ}$ of flexion. This position leads to the maximum force to be applied for a maximum duration. The head is slightly bent with an angle of between 15 and $45^{\circ}$.

Through this study literature, we find that the virtual reality technology plays an important role in many areas. In particular, the applications of VR technique in surgical simulation have been developed to provide better and better ergonomic solutions which satisfy users. Through these studies, we can better consider the virtual reality room and design the components that give a better immersion for the surgeons. Thus, we can improve the ergonomics in surgical simulation by changing haptic interface, the position of the surgeon and his posture.

\section{Related works}

\subsection{The surgeon posture}

To perform their operations with haptic sensation as in real surgical environment, we adapted and modified the haptic interfaces as well as the position of surgeon:

- Setting the table height corresponding to the surgeon's height. We chose table height is equal to 0.49 of surgeon's height. [14]. Notice that it is now possible to adjust at real time the position of table?

- Adjusting the distance between the screen and the surgeon's position. Normally, this distance is $0.60 \mathrm{~m}$, but with the giant screen in Virtual Reality room at our laboratory; we chose the distance of $1.5 \mathrm{~m}$.

- Changing position as well as the posture of the surgeon: the surgery is always in front of the screen, the direction of movement of the tool should be parallel with the spine. 


\subsection{The new Human Machine Interface}

The practitioner manipulated the haptic arm using the 3D-prining machine handle (figure 2). The position of the physician was not comparable to the real operating room environment and the conditions of experimentation not ideal:

- The surgeon was not in front of the screen and the posture position not comfortable.

- The 3D-printing handle material was different than the final product's one.

Moreover, the idea is to use the same surgical instrument on mannequin in operating room and during the simulation.

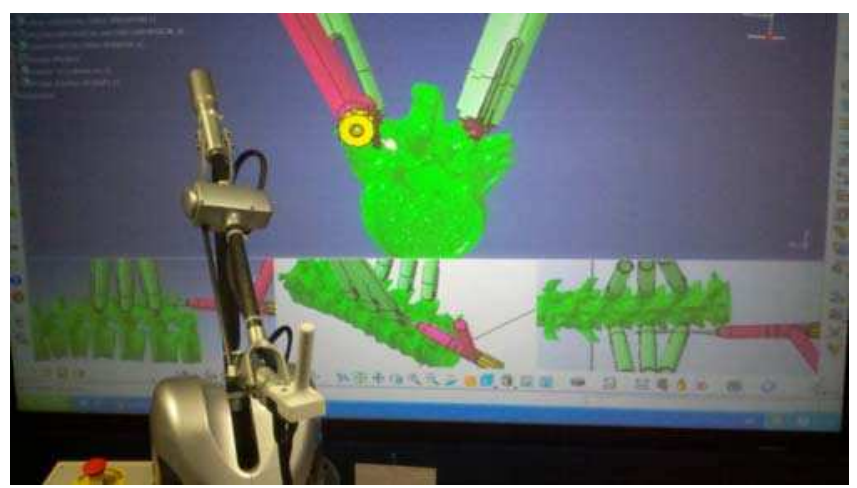

Fig. 2. Picture of the 3D-prining machine handle at the end of the haptic arm

Through ergonomics studies, we can better consider the virtual reality room and design the components that can make a better immersion of the practitioner. Thus, we can improve ergonomics in surgery simulation by changing haptic interfaces to adapt with the position and posture of the surgeon. In order that the surgeon can use the haptic arm in the operating simulation as in reality, we thought to create an intermediate mechanical piece to hang the surgical instrument prototype (Protige) at the end of the haptic arm. The objectives of this adaptation are to give the surgeon a real sensation when holding the real instrument Protige and then to carry it in a direction parallel to the spine's main axis.

Before the mechanic piece was fabricated, we carried out a numerical simulation to ensure the strength, deformation and constraints of the piece to work properly when it tightened the surgical instrument. We divided the simulation into two cases:

- Test the strength of the piece under the Protige's effort when the simulator is running maximum the instrument.

- Test the tightness of the piece under the load of the screws so it could tight well Protige.

The intermediate piece of aluminum has been made at our workshop by the Numerical Control of Machine Tools (figure 3). We observe the $90^{\circ}$ modification orientation compared to the previous 3D-prining machine handle (figure 2) 


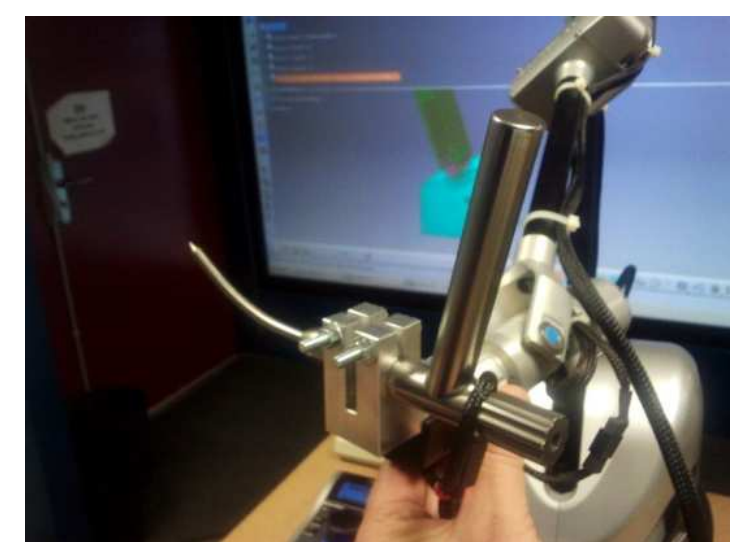

Fig. 3. The instrument tightened by the piece is tied to the haptic arm

\subsection{Testing the haptic sensation in the insertion of the rod with screw holes}

Concerning the simulations, the previous virtual surgical instruments dimensions never allows the insertion of the rod inside the pedicle screws head. The main objective of this test is to find optimal dimensions of the virtual surgical instruments and verify the friction sensation when inserting the rod into the holes of the pedicle screws head. Using a simplified virtual model, we test different manipulation situations (figure 4): changing screws holes diameters and rod diameters. We modified the diameter of the pedicle screw hole from 6 to $9 \mathrm{~mm}$. We also used different rod diameters: from 4 to $6 \mathrm{~mm}$.

To simplify the simulation with many different cases, we proposed a simple model of vertebral column and screws. Of course, the positional parameters between the screws and spine are respected as the MIS procedure. The new versions of models vertebral column and the screws are created using CATIA software.

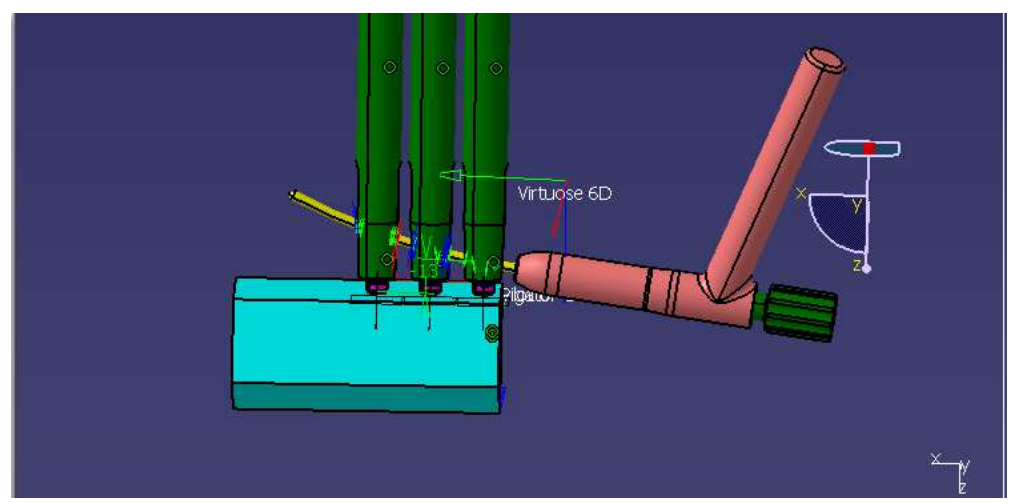

Fig. 4. The translation of Protige parallel with the spine position

We ran several simulations with different views ("multiple views" on IFC CATIA software). So we set up a kind of viewpoints allowing the surgeon to possess more isometric views, each vignette characterizing a different spatial view. 
User moves the virtual instrument over the spine and we set the test duration up to one minute to validate the feasibility of the procedure phase. Five trials are conducted for each case. The experimental duration for each case were taken in order to determine the levels of difficulty in inserting the rod into the holes. A test was considered successful if the positioning time of the rod through the holes did not exceed one minute.

We got the test results with different cases (see an extraction of the results in table 1). Simulation is recorded by video to analyze the results as well as confirmation of results.

Table 1. Results of the trials (in duration time)

\begin{tabular}{|c|c|c|c|c|c|c|c|c|c|c|c|c|}
\hline $\begin{array}{c}\text { Rod diameter } \\
(\mathrm{mm})\end{array}$ & \multicolumn{4}{|c|}{$\begin{array}{c}\text { Duration of the trials (s) } \\
\text { Screws' hole: } 8 \mathrm{~mm}\end{array}$} & $\begin{array}{c}\text { Mean } \\
(\mathrm{s})\end{array}$ & \multicolumn{5}{c|}{$\begin{array}{c}\text { Duration of the trials (s), } \\
\text { Screws' hole: } 9 \mathrm{~mm}\end{array}$} & $\begin{array}{c}\text { Mean } \\
(\mathrm{s})\end{array}$ \\
\hline 4 & 8 & 7 & 6 & 4 & 10 & 7 & 12 & 8 & 7 & 4 & 7 & 7,6 \\
\hline 4,5 & 60 & 50 & 120 & 90 & 60 & 76 & 5 & 7 & 8 & 8 & 9 & 7,4 \\
\hline 5 & & & & & & & 5 & 8 & 9 & 8 & 7 & 7,4 \\
\hline 5,5 & & & & & & & 10 & 8 & 8 & 11 & 9 & 9,2 \\
\hline
\end{tabular}

We have tested a maximum of the possible experimental conditions. Depending of the trauma cases, the physician has to use two or more screws inside the body. We asked the user to test the virtual insertion of the rod in 1, 2 and 3 screws. An extraction of the complete results is presented in table 2 .

Table 2. Conclusion for one specific configuration: extraction of the complete table. Hole of the screws: $8 \mathrm{~mm} /$ diameter of the rod 4 and $4,5 \mathrm{~mm}$

\begin{tabular}{|c|c|c|c|c|c|c|c|c|c|}
\hline \multirow{2}{*}{$\begin{array}{c}\text { Rod diameter } \\
(\mathrm{mm})\end{array}$} & \multicolumn{4}{|c|}{ Number of screws } & \multicolumn{5}{c|}{ Duration of the trials (s) } \\
\cline { 2 - 12 } & 1 & 2 & 3 & 1 & 2 & 3 & 4 & 5 & Mean (s) \\
\hline 4 & OK & OK & OK & 8 & 7 & 6 & 4 & 10 & 7 \\
\hline 4.5 & diff. & imp. & imp. & 60 & 90 & 120 & 50 & 60 & 76 \\
\hline
\end{tabular}

"OK" means that the corresponding experiment is working well. For example, inserting a $4 \mathrm{~mm}$ diameter rod through 3 pedicle screws' holes of $8 \mathrm{~mm}$ takes less than 1 minute. Inserting a $4.5 \mathrm{~mm}$ diameter rod through 1 pedicle screws' hole of $8 \mathrm{~mm}$ takes around 1 minute. We qualified this situation as difficult (diff.). Finally, it is impossible for the user to insert the $4.5 \mathrm{~mm}$ diameter rod through 2 or more pedicle screw holes of $8 \mathrm{~mm}$.

The complete experiment shows that the $9 \mathrm{~mm}$ pedicle screws' holes always allow the insertion of the rod from $4 \mathrm{~mm}$ to $5.5 \mathrm{~mm}$. For the $8 \mathrm{~mm}$ pedicle screws' holes, they are compatible with the $4 \mathrm{~mm}$ rod diameter (insertion through 3 screws) and $4.5 \mathrm{~mm}$ rod diameter (insertion through $1 \mathrm{screw}$ ). One of the reasons that prevent this insertion is the precision of the collision detection between parts using the IFC CATIA software coupled with the haptic device. It doesn't allow the relative movements be- 
tween rod and holes even if the rod's diameter is smaller than screws' holes. Moveover, the durations of the trials depend of the user's experience.

\section{Conclusion}

In this study, we not only propose a better ergonomic situation of the physician in front of the operating screen, but also increase the performance of the simulator in order to allow the manipulation of the real innovative surgical instrument developed.

We used virtual reality environment and the manufactured prototype with the aim to validate the new surgical procedure and the innovative designed surgical instrument. For that, an adaptation piece has been designed, manufactured and manipulated. This adaptation has really increased the real sensation of the user in front of the virtual reality screen.

Moreover, the disposition of the experimental room and the user has evolved. The modification of the model and the different trials with different users allow researchers to find parameters which influence the quality of physical sensation. This activity will allow

- Designers to propose tools and models more realistic for effective simulations during the design process. In consequences, design choices can be more precise

- Physicians to quickly evaluate and validate an adapted operative procedure.

These experiments with users and researchers give us some qualitative results. The next step will be the evaluation of the complete virtual environment (with different dimensional models) with numerous expert surgeons to:

- validate the design of the surgical instrument

- quantify the sensations of the experts

The surgical instruments developed are generally composed of multiple mobile parts. One of the future objectives will be to work on the possibility to manipulate all the parts of the product in virtual reality. This objective imposes the integration of multiple cameras and markers in the experimental room.

\section{References}

1. Melton GB., 2010, Biomedical and health informatics for surgery, Advanced Surgery, 44:117-30.

2. NF EN ISO 9241-210: 'Human-centred Design Processes for Interactive Systems' Genève, Switzerland, International Organization for Standardization, January, 2011.

3. Jokela, T., "Making user-centred design common sense: striving for an unambiguous and communicative UCD process model", Proceedings of the second Nordic conference on Human computer interaction. Aarhus, Denmark, ACM Press, 2002.

4. Guiatni M., Riboulet V. and Kheddar A., 2009, Design and Evaluation of a Haptic Interface for Interactive Simulation of Minimally-Invasive Surgeries, IEEE/ASME International Conference on Advanced Intelligent Mechatronics, Suntec Convention and Exhibition Center, Singapore, July 14-17, 2009. 
5. Saupin G., Duriez C., Cotin S., 2008, Contact model for haptic medical simulations, Lecture Notes In Computer Science 2008, p.157-165, Vol. 5104, Proceedings of the 4th international symposium on Biomedical Simulation, London, UK.

6. Zarrad W., Poignet P., Cortesão R. and Company O., 2007, Stability and Transparency Analysis of a Haptic Feedback Controller for Medical Applications, Proceedings of the 46th IEEE, Conference on Decision and Control, New Orleans, LA, USA, Dec. 12-14, 2007.

7. Mizokamit R., Abet N., Kinoshitatt Y., He S., 2007, Simulation of ICSI Procedure Using Virtual Haptic Feedback Model, 2007 IEEE/ICME International Conference on Complex Medical Engineering.

8. Matern, U., Waller, P., Giebmeyer, C., Rückauer, K.D., Farthmann, E.H., 2001. Ergonomics: requirements for adjusting the height of laparoscopic operating tables.JSLS: Journal of the Society of Laparoendoscopic Surgeons 5, 7e12.

9. Burgess-Limerick, R., Mon-Williams, M., Coppard, V.L., 2000. Visual display height.Human Factors: The Journal of the Human Factors and Ergonomics Society 42(1), $140 \mathrm{e} 150$.

10. Jaschinski, W., Heuer, H., Kylian, H., 1998. Preferred position of visual displays relative to the eyes: a field study of visual strain and individual differences. Ergonomics 41 (7), $1034 \mathrm{e} 1049]$

11. Turville, K.L., Psihogios, J.P., Ulmer, T.R., Mirka, G.A., 1998. The effects of video display terminal height on the operator: a comparison of the $15^{\circ}$ and $40^{\circ}$ recommendations. Applied Ergonomics 29 (4), 239e246.

12. Van Veelen, M.A., Kazemier, G., Koopman, J., Goossens, R.H., Miejer, D.W., 2002. Assessment of the ergonomically optimal operating surface height for laparoscopic surgery. Journal of Laparoendoscopic \& Advanced Surgical Techniques 12 (1), 47e52.

13. Gurvinder Kaur :Role of OT Table Height on the Task Performance of Minimal Access Surgery World Journal of Laparoscopic Surgery, January-April 2008;1(1):49-55

14. Van Veelen, M.A., 2003. Human-Product Interaction in Minimally Invasive Surgery: A Design Vision for Innovative Products. Delft University of Technology, Delft,the Netherlands. pp. 92e97.

15. Matern, U., Waller, P., 1999. Instruments for minimally invasive surgery. Surgical Endoscopy 13 (2), 174e182. 